\title{
Bertrand Russell Sobre a Matemática nos PrinCíPIOS
}

\author{
John A. Fossa \\ Universidade Estadual da Paraíba - UEPB - Brasil
}

(aceito para publicação em agosto de 2021)

\begin{abstract}
Resumo
O presente trabalho faz uma breve análise do logicismo de Bertrand Russell, focando em especial nas suas implicações para a natureza da matemática. Conclui-se que o maior legado do logicismo é uma apresentação sistemática da unidade da matemática. Em anexo, há uma tradução do Capítulo 1 de The Principles of Mathematics.
\end{abstract}

Palavras-chave: História da Matemática, Logicismo, Bertrand Russell.

\section{[Bertrand Russell on Mathematics in The Principles]}

\begin{abstract}
The present work is a brief analysis of Bertrand Russell's logicism, especially as it relates to the nature of mathematics. It is concluded that logicism's greatest legacy is a systematic presentation of the unity of mathematics. A Portuguese translation of Chapter 1 of The Principles of Mathematics is annexed.
\end{abstract}

Keywords: History of Mathematics, Logicism, Bertrand Russell.

\section{Introdução}

Segundo Immanuel Kant (1966, p. 23), “A Matemática e a Física são as duas ciências teóricas, cujos objetos devem ser dados a priori, a primeira de forma completamente 
pura, ...”1 Continua por caracterizar a matemática como tendo certeza a priori. De fato, o projeto fundamental da Crítica de Kant é o de mostrar que a matemática é sintética $a$ priori. Sendo sintética, como oposto a analítica, a matemática nos fornece conhecimento novo. Sendo a priori, a matemática nos fornece conhecimento certo. Será este segundo aspecto a certeza da matemática - que será mais importante para o presente trabalho.

\section{Algumas Raízes históricas}

Como se sabe, Kant não foi o primeiro pensador a enaltecer a certeza da matemática e nem foi o último. ${ }^{2}$ De fato, era a Academia de Platão que foi o, por assim dizer, locus classicus da tese da confiabilidade do conhecimento matemático. Para garantir a verdade de proposições matematicas, se inventou, na referida Academia, a metodologia axiomática conforme explicada, por exemplo, em Lasserre (1966). ${ }^{3}$ O método foi perfeiçoado e publicado por Aristóteles enquanto ele era ainda membro da Academia.

Os Elementos de Euclides, um aluno de Aristóteles, são uma aplicação magistral do método à geometria e o sucesso dessa obra durante os séculos seguintes sem dúvida contribuiu para a propagação da conceituação da matemática como conhecimento certo. Na verdade, a grande controvérsia sobre o quinto postulo de Euclides só vem a confirmar a importância da referida tese. Conforme a explanação de Fossa (2012), o quinto postulado não era considerado intuitivamente óbvio e assim comprometia a confiabilidade de quase toda a geometria. Para retificar a situação era necessário ou substitui-lo com outro postulado com o mesmo poder gerativo ou demonstrá-lo como um teorema. No entanto, todas as duas alternativos fracaçaram.

Eventualmente a independência do quinto postulado foi estabelecida e geometrias não-euclidianas foram desenvolvidas. ${ }^{4}$ Isso, porém, foi altamente indesejável, pois implicava que haja duas (ou mais) geometrias incompatáveis que nos fornecem verdades absolutas sobre o espaço físico.

Ao mesmo tempo, desenvolvimentos na então recém-criada Teoria dos Conjuntos levaram os matemáticos a situações que eram não somente anti-intuitivas, mas também contraditórias. ${ }^{5}$ Esse resultado era potencialmente até mais preocupante do que a situação problemática da geometria. Mas, em todo caso, a eclosão dos dois grupos de dificuldades, mais ou menos simultaneamente, abalou a confiaça outroramente depositada na matemática.

Para tentar recuperar o status privilegiado da matemática, sugeram-se três importantes teorias sobre a natureza da matemática, viz, o formalismo de David Hilbert (1862-1943), o intuitionismo de L. E. J. Brouwer (1881-1966), e o logicismo de Gottlob

\footnotetext{
${ }^{1} \mathrm{M}$ a t h e m a t i k und P h y s i k sind die beiden theoretischen Erkenntnisse der Vernuft, welche ihre O b j e k t e a priori bestimmen sollen, die erstere ganz rein, ...

A tradução dada no texto é nossa.

${ }^{2}$ A tese da certeza matemática é hodiernamente controversa. Kitcher (1984), por exemplo, é um dos primeiros importantes obras defensoras do falibilismo na matemática.

${ }^{3}$ Ver também a edição ampliada Lasserre (1990).

${ }^{4}$ Para mais detalhes, ver, por exemplo, Meschkowski (1965).

${ }^{5}$ De novo, Meschkowski (1965) pode ser consultado para mais detalhes.
} 
Frege (1848-1925) e Bertrand Russell (1872-1970), ${ }^{6}$ sendo esse último o objeto do nosso interesse aqui.

\section{Bertrand Russell}

Embora Russell seja bastante conhecido da maioria dos leitores da presente revista, será oportuno, mesmo assim, traçar algumas linhas rápidas sobre a sua biografia, para o qual nos valeremos das informações contidas em Irvine (1996).

Nascido na casa do campo dos seus pais, no País de Gaules, à uma família da "nobreza" britânica, Russell, ainda criança, veio a morar com os avôs paternos devido ao falecimento dos seus progenitores. Foi educado por tutores particulares até entrou em Trinity College, Cambridge, onde cursou ambos matemática e filosofia moral. Como matemático, contribuiu aos fundamentos da matemática e à lógica formal. Como filósofo é mais lembrado para as suas contribuições à filosofia analítica e ao pacifismo.

Devido ao seus trabalhos matemáticos, Russell foi admitido à Sociedade Real em 1908. Em 1932, ganhou a Medalha De Morgan da Sociedade Matemática de Londres e, em 1934, a Medalha Sylvester da Sociedade Real. Enquanto Russell ganhou muita fama como matemático, sua aderência ao pacifismo lhe rendeu certo opróbrio. Foi demitido da sua posição na sua alma mater em 1916, preso por seis meses em 1918 e, em 1940, demitido de uma posição na City College de New York (agora parte da CUNY). De fato, seu posicionamento não era bem visto durante as duas Guerras Mundiais, mas se tornaria mais aceito durante a época da Guerra de Vietnã. Além de se opor à guerra de forma geral, apoiava as lutas contra a discriminação contra mulheres e contra discriminação racial. Foi também um dos fundadores da influente Conferência Pugwash, instituída originalmente para lutar contra a proliferação de armas nucleares. Enfim, as suas atividades pacifistas receberam aprovação oblíqua quando Russell ganhou o Prêmio Nobel em Literatura em 1950.

\section{Os Principles}

Os Princípios da Matemática (The Principles of Mathematics) foram publicados em 1903. A publicação havia sida preterida por uns dois anos, enquanto Russell lutava com um paradoxo, batizado com seu próprio nome, na Teoria dos Conjuntos. Era nessa época que Russell começou a colaborar com seu colega e outrora professor, Alfred North Whitehead (1861-1947). Os dois haviam conhecido o trabalho de Giuseppe Peano (1858-1932) sobre os fundamentos da matemática quando foram para o Congresso Internacional dos Matemáticos de Paris em $1900{ }^{8}$ Russell só iria conhecer o importante trabalho de Frege posteriormente. A colaboração inicial com Whitehead visava a produção de um segundo volume dos Princípios. Ficou logo evidente para os dois, contudo, que era necessário uma

\footnotetext{
${ }^{6}$ Ver, por exmplo, Da Costa (1992). Há várias edições mais recentes dessa obra.

${ }^{7}$ O presente autor acha o sistema do royality inerentemente nojento, mas reconhece a nobreza de espírito e as realizações de Bertrand Russell.

${ }^{8}$ Ver O’Connor e Robertson (2003).
} 
obra independente, que completasse sim os Princípios, mas que seria mais formal e mais rigorosa do ponto de vista matemático. ${ }^{9} \mathrm{O}$ resultado foi os Principia Mathemática (Princípios Matemáticos), publicados em três volumes, originalmente nos anos de 1910, 1912 e 1913. Os Principia também incorporaram o trabalho de Frege.

Tanto os Príncípios, quanto os Principia, visavam a resolução dos problemas que haviam surgidos referente à certeza do conhecimento matemático por reduzir a matemática à lógica. Visto que a lógica é uma teoria mais simples do que a matemática e já que os seus primeiros princípios são mais intuitivamente aceitáveis do que os da matemática, a proposta redução daria um fundamento mais seguro à própria matemática. Para efetuar a referida redução era necessário fazer as seguintes duas tarefas: definir os termos matemáticos em termos dos da lógica e demonstrar os teoremas da matemática a partir dos da lógica. Para tanto, Russell e Whitehead conceberam a lógica como contendo a Teoria dos Conjuntos.

\section{A Natureza hipotética da matemática}

A visão de Russell, de que a matemática se reduz à lógica, é chamado de logicismo. Ela permiteu ao Russell definir, ou pelo menos caracterizar, a matemática como um certo subconjunto da classe de proposições ${ }^{10}$ da forma " $p$ implica $q$ ", onde $p$ e $q$ são proposições (ver a tradução apresentada ao final dos presentes comentários). A palavra "implicar" tem duas acepções distintas, mas relacionadas. A primeira significa a relação entre a(s) premissa(s) e a conclusão de um argumento válido e pode ser representada da seguinte forma:

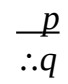

Aqui $p$ é a premissa do argumento; é separada da conclusão, $q$, por um traço. O símbolo $\therefore$ quer dizer "portanto". O argumento afirma que a conclusão q é uma consequência lógica da premissa $p$. Exprime-se isso frequentemente pela simbolização $p \vdash q$.

A segunda acepção da palavra "implicar" é a expressa pela proposição "se $p$, então $q$ ". Aqui $p$ é o "antecedente" e $q$ o "consequente" da proposição. A proposição toda é chamada de condicional. Na lógica, o condicional é concebido como uma constante lógica. É simbolizada em várias maneiras, sendo $p \supset q, p \rightarrow q$ e $p \Rightarrow q$ entre as mais comuns. A referida constante é entendida como sendo verdade-funcional. Isto é, a sua verdade ou falsidade é determinada pela verdade e falsidade dos seus componentes sentenciais, ou seja, do antecedente $p$ e consequente $q$. Por considerar todas as possibilidades de verdade e falsidade dos componentes, o significado do condicional pode ser exibido através da seguinte tabela:

\footnotetext{
${ }^{9}$ Ver o Prefácio dos Principia (Whitehead and Russell, 1910).

${ }^{10}$ Russell permite que fórmulas contendo variáveis abertas sejam proposições.
} 


\begin{tabular}{|c|c|c|}
\hline$p$ & $q$ & $p \rightarrow q$ \\
\hline $\mathrm{V}$ & $\mathrm{V}$ & $\mathrm{V}$ \\
\hline $\mathrm{V}$ & $\mathrm{F}$ & $\mathrm{F}$ \\
\hline $\mathrm{F}$ & $\mathrm{V}$ & $\mathrm{V}$ \\
\hline $\mathrm{F}$ & $\mathrm{F}$ & $\mathrm{V}$ \\
\hline
\end{tabular}

onde, obviamente, V e F indicam, respectivamente, "verdadeiro" e "falso".

Uma olhada atenta à referida tabela mostrará que o condicional sempre será verdadeiro se o seu antecedente for falso ("uma mentira implica em qualquer coisa") ou se seu consequente for verdadeiro ("a verdade é implicada por qualquer coisa"). Essas são as assim chamados paradoxos da "implicação material” ou "condicional material”, como essa constante é chamada para diferenciá-la de outros tipos de condicionais, os da lógica modal e da lógica relevante, por exemplo. Não obstante a existência desses "paradoxos", a lógica baseada no condicional material é o substrato lógico mais usada na matemática. (Isso não é problemático!)

No trecho dos Princípios traduzido abaixo, Russell usa a palavra "implica” sempre na segunda acepção, a do condicional material. Como foi indicado no parágrafo anterior, isto é o procedimento operacional padrão na matemática. Em vez de "antecedente”, ele usa "hipótese”. Assim, segundo Russell, a forma básica de proposições matemáticas é $h \supset c$, onde $\supset$ é o condicional material, $h$ é sua hipótese, ou antecedente, e $c$ é o seu consequente.

\section{Dois Exemplos}

Será instrutivo, nesse ponto, olhar alguns exemplos. Escolhemos dois exemplos de um livro de Elon Lages Lima, pois ele é considerado um exímio escritor de prosa matemática. O primeiro exemplo é Teorema 1 do Capítulo VIII do seu Curso de Análise, Lima (1987, p. 205):

Se existe a derivada $f^{\prime}(a)$ então $f$ é contínua no ponto $a$.

O referido Teorema 1 é claramente um exemplo da forma exigida por Russell, pois, pondo

$$
\begin{gathered}
h=a \text { derivada } \vdash \text { existe } \\
c=f \text { é contínua no ponto } a,
\end{gathered}
$$

a sua forma simbolica é

$$
p \Rightarrow q .
$$

O Teorema 3 (Regra da cadeia) do mesmo capítulo, no entanto, é mais complicado. Temos, em Lima (1987, p. 206), 
John A. Fossa

$$
\begin{aligned}
& \text { Sejam } f: X \rightarrow R, \quad g: Y \rightarrow R, \quad F(X) \subset Y, \quad a \in X \cap X^{\prime} \text {, } \\
& b=f^{\prime}(a) . \quad \text { Se existem } f^{\prime}(a) \text { e } p \supset q \text { então } \\
& g \circ f: X \rightarrow R \quad \text { é derivável no ponto } a \text {, valendo } \\
& (g \circ f)^{\prime}(a)=g^{\prime}(b) \cdot f^{\prime}(a) \text {. }
\end{aligned}
$$

A forma desse teorema é bastante mais complexo. Nem parece uma proposição, mas sim um argumento, em que a primeira sentença lista várias condições. A conclusão tem a forma de um condicional, mas não um condicional simples, pois tanto o antecedente, quanto o consequente tem partes sentenciais. Observamos que a seta $(\rightarrow)$ que aparece no teorema não representa o condicional, mas pode ser lido como “em”, fazendo parte da expressão “ $f$ é uma função de $X$ em $R$ ", etc.

Ora, visto que "condição" é sinônimo de "hipótese”, podemos fazer a seguinte interpretação do teorema, pondo

$$
\begin{array}{ll}
h_{1}=f: X \rightarrow R & h_{5}=(b=f(a)) \\
h_{2}=g: Y \rightarrow R & h_{6}=f^{\prime}(a) \text { existe } \\
h_{3}=F(X) \subset Y & h_{7}=g^{\prime}(b) \text { existe } \\
h_{4}=a \in X \cap X^{\prime} & \\
c_{1}=g \circ f: X \rightarrow R \text { é derivável no ponto } a \\
c_{2}=\left[(g \circ f)^{\prime}(a)=g^{\prime}(b) \cdot f^{\prime}(a)\right] .
\end{array}
$$

Assim, o teorema tem a forma de um argumento com a forma

$$
\begin{aligned}
& h_{1} \\
& h_{2} \\
& h_{3} \\
& h_{4} \\
& \underline{h}_{5} \\
& \therefore\left(h_{6} \& h_{7}\right) \supset\left(c_{1} \& c_{2}\right)
\end{aligned}
$$

Na formulação o símbolo \& representa a conjunção “e” (uma constante lógica). Ainda podemos escrever o argumento de forma linear, nos valendo do símbolo $h \ni c$, da seguinte maneira:

$$
h_{1}, h_{2}, h_{3}, h_{4}, h_{5} \vdash\left(h_{6} \wedge h_{7}\right) \supset\left(c_{1} \wedge c_{2}\right) .
$$

Para ver que o resultado aqui obtido é nada mais do que uma variante estilística da forma de Russell, precisamos voltar ao fato já mencionado de que as duas acepções de "implicar”, a de consequência lógica e a de condicional material, são relacionadas. A relação é dada pelo Teorema da Dedução, que se obtém na lógica subjacente para a matemática. Expresso simbolicamente, temos 


$$
h \vdash c \text { se e somente se } h \supset c^{11}
$$

Isto é, podemos converter uma premissa de um argumento num antecedente de um condicional, e vice versa.

Desta forma, o argumento do citado Teorema 3 pode ser express como

$$
h_{1}, h_{2}, h_{3}, h_{4}, h_{5},\left(h_{6} \wedge h_{7}\right) \vdash\left(c_{1} \wedge c_{2}\right) .
$$

Agora podemos juntar todas as condições em uma só premissa, usando a conjunção “e”, obtendo assim

$$
\left(h \| 1 \wedge h_{2} \wedge h_{3} \wedge h_{4} \wedge h_{5} \wedge h_{6} \wedge h_{7}\right) \vdash\left(c_{1} \wedge c_{2}\right) .
$$

Utilizando o Teorema da Dedução mais uma vez, obtemos

$$
\left(h \| 1 \wedge h_{2} \wedge h_{3} \wedge h_{4} \wedge h_{5} \wedge h_{6} \wedge h_{7}\right) \supset\left(c_{1} \wedge c_{2}\right) .
$$

Finalmente, ao por

$$
\begin{aligned}
& h= \\
& c=c_{1} \wedge c_{2}
\end{aligned}
$$

obtemos a forma de Russell

$$
h \supset c .
$$

Obviamente, os exemplos não mostram que toda proposição matemática tem a forma de Russell, mas indicam como sua afirmação deve ser entendida. Enquanto sua forma básica deve ser a de um condicional, os seus componentes, o antecedente e o consequente, podem ter composições complexas. Há, no entanto, certas limitações sobre a composição interna dos referidos componentes no que se diz referente às variáves e constantes neles contidas. Isto é explicado no texto de Russell traduzido abaixo.

\section{Pequena Reflexão sobre o projeto de Russell}

Como já indicamos, o logicismo de Russell era uma tentativa de resgatar a certeza da matemática dos abalos que ela sofreu nos séculos 18 e 19. Para fazer a sua proposta redução da matemática à lógica, contudo, era necessário introduzir ao formalismo vários axiomas técnicos nada intuitivos e isso comprometeu a obtenção do resultado desejado. Não entraremos nos detalhes técnicas aqui.

\footnotetext{
${ }^{11}$ Mais propriamente, $h \vdash c$ se e somente se $\vdash h \supset c$. Mais geralmente, $\Sigma, h \vdash c$ se e somente se $\Sigma b=f^{\prime}(a)$ , onde $\Sigma$ é um conjunto de proposições.
}

RBHM, Vol. 21, nº 42, pp. 329-349, 2021 
Há outra crítica, porém, que poderemos discutir brevemente, pois esclarece o próprio projeto do logicismo. Trata-se do julgamento crítico de que Russell não reduziu a matemática à lógica, mas à lógica e à Teoria dos Conjuntos. Considero essa crítica um tanto mesquinha. Explico a seguir.

A certeza, a menos de uma pálida sombra dela na forma de certeza psicológica, não é dada ao homem. Isso acontece porque o todo se relaciona com cada parte e cada parte com o todo, mas devido à finitude do homem e à natureza linear do seu raciocínio, não podemos abarcar a totalidade dessas relações. Isso não significa, porém, que não devemos alcançar o mais que podemos da certeza. Nesse sentido, o matemático, como os heróis dos sagas nórdicas que sairam à luta sabendo que não poderiam vencer, almeja sempre mais e mais certeza através do rigor dos seus argumentos, mesmo que não a pode alcançar plenamente.

Dado esse contexto, uma apreciação mais acertada do trabalho de Russell deverá se centrar no fato de que a redução por ele proposta mostra de forma magistral a unidade da matemática. De fato, vislumbramos essa unidade nos frequentes cruzamentos de subáreas da matemática em que resultados de uma área são aplicáveis, de forma surpreendente, a outra área aparentemente distinta. Isso foi reconhecido, por exemplo, pelo "programa Langlands”, instituído por Robert Langlands (1936-) nos meados da década de 1960. Foi o trabalho de Russell, porém, que mostrou, de forma sistemática, a vasta unidade entre todas as áreas da matemática.

\section{Referências}

DA COSTA, Newton. 1992. Introdução aos fundamentos da matemática. São Paulo: EDUSP.

FOSSA, John A. 2012. O que há de errado com o quinto postulado de Euclides? In: FOSSA, John A. Ensaios sobre a Educação Matemática. São Paulo: Editora Livraria da Física.

IRVINE, A. D. 1996. Bertrand Arthur William Russell. Disponível em https://mathshistory.st-andrews.ac.uk/Biographies/Russell/. Acesso: 02/03/2021.

KANT, Immanuel. 1966. Kritik der reinen Vernuft. Stuttgart: Philipp Reclam.

KITCHER, Philip. 1984. The Nature of Mathematical Knowledge. Oxford: Oxford University Press.

LASSERRE, François. 1990. La naissance des mathématiques à l'époque de Platon. Paris: Éditions du Cerf.

LASSERRE, François. 1966. The Birth of Mathematics in the Age of Plato. Cleveland: Meridian Books.

LIMA, Elon Lages. 1987. Curso de análise. Vol. 1. Rio de Janeiro: IMPA. 
MESCHKOWSKI, Herbert. 1965. Evolution of Mathematical Thought. San Francisco: Holden-Day.

O’CONNOR, J. J., e ROBERTSON, E. F. 2003. Alfred North Whitehead. Disponível em https://mathshistory.st-andrews.ac.uk/Biographies/Whitehead/. Acesso: 02/03/2021.

WHITEHEAD, Alfred North, \& RUSSELL, Bertrand. 1910. Principia Mathematica. Vol. I. Cambridge: University of Cambridge Press.

\section{John A. Fossa}

Programa de Pós-Graduação em Ensino de Ciências e Educação Matemática - PPGECM - da UEPB, campus de Campina Grande - Brasil

E-mail: jfossa03@gmail.com 


\title{
Anexo I
}

[De The Principles of Mathematics por Bertrand Russell]

\author{
CAPÍTULO I \\ DEFINIÇÃO DE MATEMÁTICA PURA
}

1. Matemática Pura é a classe de todas as proposições da forma " $p$ implica $q$ ”, onde $p$ e $q$ são proposições contendo uma ou mais variáveis, as mesmas nas duas proposições, e [nas quais] nem $p$ nem $q$ contêm qualquer constante exceto constantes lógicas. E constantes lógicas são todas as noções definíveis em termos das seguintes: Implicação, a relação de um termo à uma classe de que é um membro, a noção de tal que, a noção de relação, e tais outras relações que possam ser envolvidas na noção geral de proposições da referida forma. Além dessas, a matemática usa uma noção que não é um componente das proposições que considera, a saber, a noção de verdade.

2. A referida definição de matemática pura é, sem dúvida, um tanto inusitada. Suas diversas partes, no entanto, parecem ser passíveis de justificação exata - uma justificação que será o objeto da presente obra. Será mostrado que tudo o que tem sido julgado ser matemática pura, no passado, é incluído na nossa definição, e que tudo a mais que seja incluído [na definição] possui as marcas pelas quais a matemática é geralmente, embora vagamente, distinguida de outros estudos. A definição não se propõe ser uma escolha arbitrária para usar uma palavra comum num sentido incomum, mas sim uma análise precisa das ideias que são supostas, mais ou menos conscientemente, no uso corriqueiro do termo. Nosso método será, portanto, um de análise, e nosso problema pode ser chamado filosófico - no sentido, isto é, de procurarmos passar do complexo para o simples, do demonstrável para as suas premissas indemonstráveis. Mas, em um aspecto, várias das nossas discussões serão diferentes das que geralmente são chamadas filosóficas. Poderemos, graças ao trabalho dos próprios matemáticos, alcançar a certeza sobre a maioria das questões que nos interessarão; e, entre as passíveis de uma solução exata, acharemos muitos dos problemas que, no passado, têm sido envolvidos em toda a incerteza da contenda filosófica. A natureza de número, de infinidade, de espaço, tempo e movimento, e da própria inferência matemática são todas questões às quais, na presente obra, serão dadas respostas que se mostram demonstráveis com certeza matemática - respostas, porém, que consistem em reduzir os referidos problemas a problemas de lógica pura; esse último [problema] não será resolvido de forma satisfatória no que segue.

3. A Filosofia da Matemática tem sido até agora tão controversa, obscura e não progressiva quanto os outros ramos da filosofia. Embora geralmente se concordasse que a matemática é, em algum sentido, verdadeira, os filósofos disputavam sobre o que as proposições matemáticas realmente significassem: embora algo fosse verdadeiro, não houve duas pessoas que concordavam sobre o que foi que fosse verdadeira, e se algo fosse conhecido, ninguém sabia o que foi que fosse conhecido. Enquanto isso era duvidoso, porém, não 
poderia ser dito que se obtivesse qualquer conhecimento certo e exato na matemática. Vemos, em consequência, que os idealistas têm se inclinado, mais e mais, a considerar toda a matemática como [algo que] lida com mera aparência, enquanto os empiristas têm sustentado que tudo que é matemático é uma aproximação a alguma verdade sobre a qual há nada que podiam nos dizer. Esse estado de coisas, deve se confessar, era completamente insatisfatório. A Filosofia indaga da Matemática: O que significa? A Matemática, no passado, não era capaz de responder, e a Filosofia respondia por introduzir a noção irrelevante de mente. Mas agora a Matemática pode responder, pelo menos ao ponto de reduzir a totalidade das suas proposições a certas noções fundamentais da lógica. Aqui, a discussão deve ser retomada pela Filosofia. Tentarei indicar as noções fundamentais envolvidas, demonstrar detalhadamente que não há outras que ocorrem na matemática, e indicar brevemente as dificuldades filosóficas envolvidas na análise dessas noções. Um tratamento completo dessas dificuldades envolveria um tratado sobre a Lógica, o que não será achado nas seguintes páginas.

4. Houve, até bem recentemente, uma notável dificuldade com os princípios da matemática. Parecia evidente que a matemática consiste em deduções, e mesmo assim todas as explanações ortodoxas da dedução eram largamente ou completamente inaplicáveis à matemática existente. Não somente a teoria silogística aristotélica, mas também as doutrinas modernas da Lógica Simbólica, eram ou teoricamente inadequadas ao raciocínio matemático, ou, em qualquer caso, requeriam formas de expressão tão artificiais que não podiam ser aplicadas na prática. Foi nesse fato que repousava a força do ponto de vista kantiano, que asseverava que o raciocínio matemático não é estritamente formal, mas sempre usa intuições, i.e., o conhecimento a priori de espaço e tempo. Graças ao progresso da Lógica Simbólica, especialmente como tratado pelo Professor Peano, essa parte da filosofia kantiana é agora passível de uma refutação definitiva e irrevogável. Com a ajuda de dez princípios de dedução e mais dez outras premissas de uma natureza lógica geral (e.g., "implicação é uma relação"), toda a matemática pode ser deduzida de forma rigorosa e formal; e todas as entidades que ocorrem na matemática podem ser definidas em termos das que ocorrem nas referidas vinte premissas. Aqui, Matemática inclui não somente a Aritmética e a Análise, mas também a Geometria, Euclidiana e Não-euclidiana, a Dinâmica racional, e um número indefinido de outros estudos ainda não nascidos ou na sua infância. O fato de que toda a Matemática é Lógica Simbólica é uma das maiores descobertas da nossa época; e quando esse fato estiver estabelecido, o resto da matemática consistirá na análise da própria Lógica Simbólica.

5. A doutrina geral de que toda a matemática é dedução por princípios lógicos de princípios lógicos foi fortemente defendida por Leibniz, quem insistia constantemente que deveria se aferir $^{12}$ axiomas e deveria se definir todas as noções, exceto algumas poucas fundamentais. Mas, devido, em parte, a uma lógica defeituosa e, em parte, a uma crença na necessidade da Geometria Euclidiana, ele foi levado a fazer erros incorrigíveis na tentativa de executar em

\footnotetext{
12 Russell usa a palavra prove, que tem duas acepções, sendo a primordial a de testar e, talvez a mais usada hodiernamente, a de demonstrar. Em se tratar de axiomas, entendemos que ele está afirmando que os axiomas devem ser testados usando tais critérios como produtividade e consistência.
}

RBHM, Vol. 21, n 42, pp. 329-349, 2021 
detalhes uma visão que, em sua concepção geral, é agora conhecida ser correta*13. As próprias proposições de Euclides, por exemplo, não se seguem somente dos princípios da lógica; e a percepção desse fato levou Kant a suas inovações na teoria do conhecimento. Mas, a partir do crescimento da Geometria não-Euclidiana, tem ficado manifesto que a matemática pura não se importa com a questão da validez, ou não, no espaço real, dos axiomas e proposições de Euclides: essa é uma questão para a matemática aplicada, a ser decidida, na medida em que uma decisão seja possível, por experimentação e observação. O que a matemática pura afirma é somente que as proposições euclidianas se seguem dos axiomas euclidianos - i.e., afirma uma implicação: qualquer espaço que tem tais e tais propriedades também tem tais e tais outras propriedades. Assim, de forma em que são tratadas da matemática pura, as Geometrias Euclidianas e não-Euclidianas são igualmente verdadeiras: em cada uma, nada é afirmado exceto implicações. Todas as proposições que rezam sobre o que realmente existe, como o espaço em que vivemos, pertencem à ciência empírica ou experimental, não à matemática; quando pertencem à matemática aplicada, surgem da atribuição, a uma, ou mais, das variáveis de uma proposição da matemática pura, algum valor constante que satisfaz a hipótese e, assim, [isso] nos permite, para este valor da variável, afirmar, de fato, ambos a hipótese e o consequente, em vez de afirmar somente a implicação. Sempre afirmamos, na matemática, que, se uma dada afirmação $p$ seja verdadeira para qualquer entidade $x$, ou para qualquer conjunto de entidades $x, y, z, \ldots$, então alguma outra afirmação $q$ é verdadeira para essas entidades; porém, não afirmamos ou $p$ ou $q$ isoladamente para as nossas entidades. Afirmamos uma relação entre as afirmações $p$ e $q$, que chamarei de implicação formal.

6. Proposições matemáticas são caracterizadas não somente pelo fato de que afirmam implicações, mas também pelo fato de que contêm variáveis. A noção da variável é uma das mais difíceis com que a Logica deve lidar e, na presente obra, uma teoria satisfatória sobre a sua natureza, embora [nela] haja muita discussão, mal será encontrada. Para o momento, somente quero esclarecer que há variáveis em todas as proposições matemáticas, mesmo quando podem parecer estar ausentes. Poderia pensar que a Aritmética Elementar seja uma exceção: $1+1=2$ parece nem conter variáveis, nem afirmar uma implicação. Mas, de fato, como será mostrado na Parte II, o verdadeiro significado dessa proposição é: "Se $x$ é um e $y$ é um, e $x$ difere de $y$, então $x$ e $y$ são dois.” E essa proposição não somente contém variáveis, mas também afirma uma implicação. Sempre encontraremos, em todas as proposições matemáticas, que as palavras qualquer ou alguns ocorrem; e essas palavras são as marcas de uma variável e uma implicação formal. Assim, a proposição acima pode ser expressada na forma: "Qualquer unidade e qualquer outra unidade são duas unidades.” A proposição típica da matemática é da forma " $\varphi(x, y, z, \ldots)$ implica $\psi(x, y, z, \ldots)$, qualquer que sejam os valores de $x, y, z, \ldots$ ”; onde $\varphi(x, y, z, \ldots)$ e $\psi(x, y, z, \ldots)$, para todo conjunto de valores para $x, y, z, \ldots$, são proposições. Não se afirma que $\varphi$ é sempre verdadeira, nem que $\psi$ é sempre verdadeira, mas simplesmente que, em todos os casos, tanto quando $\varphi$ é falso, bem quando $\varphi$ é verdadeiro, $\psi$ se segue dele.

\footnotetext{
${ }^{13}$ Nota de Russell: *Sobre isso, cf. Couturat, La Logique de Leibniz, Paris, 1901.
} 
A diferença entre uma variável e uma constante é obscurecida um tanto pelo uso matemático. É usual, por exemplo, falar de parâmetros como sendo, em algum sentido, constantes, mas esse é um uso que temos de rejeitar. Uma constante deve ser algo que é absolutamente definido, sobre o que não há ambiguidade alguma. Assim, 1, 2, 3, e, $\pi$, Sócrates são constantes; assim são também homem, e a raça humana, passada, presente e futura, considerada coletivamente. Proposição, implicação, classe, etc., são constantes; mas uma proposição, qualquer proposição, alguma proposição não são constantes, pois essas frases não denotam um só delimitado objeto. E, portanto, o que são chamados de parâmetros são simplesmente variáveis. Veja, por exemplo, a equação $a x+b y+c=0$, considerada como a equação de uma reta num plano. Aqui dizemos que $x$ e $y$ são variáveis, enquanto $a, b, c$ são constantes. Exceto, porém, se estivermos abordando uma absolutamente determinada reta, digamos a reta de um ponto específico de Londres a um ponto específico de Cambridge, nossos $a, b, c$ não são números determinados, mas representam quaisquer números e, assim, também são variáveis. E, em Geometria, ninguém lida com linhas reais determinadas; sempre discutimos qualquer linha. $\mathrm{O}$ ponto é que colecionamos os vários pares $x, y$ em classes de classes, sendo cada classe definida como aqueles pares que têm uma determinada relação fixa a uma tríade $(a, b, c)$. Mas, de classe para classe, $a, b, c$ também variam e são, portanto, propriamente variáveis.

7. É usual, na matemática, considerar as nossas variáveis como restritas a certas classes: na Aritmética, por exemplo, é suposto que representam números. Mas, isso só quer dizer que se representam números, satisfazem alguma fórmula, i.e., a hipótese que são números implica a fórmula. É isso, então, que é realmente afirmado e, na referida proposição, não é mais necessário que as nossas variáveis sejam números: a implicação é igualmente verdadeira quando não os são. Assim, por exemplo, a proposição " $x$ e $y$ são números implica que $(x+y)^{2}=x^{2}+2 x y+y^{2}$ " é igualmente verdadeiro se, por $x$ e $y$, substituímos Sócrates e Platão ${ }^{14}$ : ambos a hipótese e o consequente, nesse caso, serão falsos, mas a implicação ainda será verdadeira. Assim, em toda proposição da matemática pura, quando elaborada de forma completa, as variáveis têm um domínio [field] absolutamente irrestrito: qualquer entidade concebível pode ser substituída por qualquer variável sem prejudicar a verdade da nossa proposição.

8. Agora podemos entender porque as constantes na matemática devem ser restritas a constantes lógicas no sentido definido acima. O processo de transformar as constantes de uma proposição em variáveis leva a que é chamado de generalização, e nos dá, por assim dizer, a essência formal de uma proposição. A matemática se interessa, exclusivamente, em tipos de proposições; se for proposta uma proposição $p$, contendo apenas constantes, e se imaginarmos substituídos, por algum dos seus termos, outros, sucessivamente, o resultado será, em geral, verdadeiro às vezes e às vezes falso. Assim, por exemplo, temos "Sócrates é um homem"; nela transformemos Sócrates numa variável e consideremos “ $x$ é um homem”. Algumas hipóteses sobre $x$, por exemplo, " $x$ é um grego", garantem a verdade de " $x$ é um homem”; assim, “ $x$ é um grego" implica " $x$ é um homem” e isso é verdadeiro para todos os

\footnotetext{
${ }^{14}$ Nota de Russell: *É necessário supor que a adição e a multiplicação aritméticas sejam definidas (como pode ser facilmente feito), para que a referida fórmula permaneça significativa quando $x$ e $y$ não são números.
}

RBHM, Vol. 21, n 42, pp. 329-349, 2021 
valores de $x$. Mas, a afirmação não pertence à matemática pura porque depende da natureza específica de grego e homem. Podemos, porém, os fazer variar também e obtemos: Se $a$ e $b$ são classes, e $a$ é contido em $b$, então " $x$ é um $a$ " implica " $x$ é um $b$ ". Aqui temos enfim uma proposição da matemática pura, contendo três variáveis e as constantes classe, contido em e as envolvidas na noção de implicações com variáveis. Na medida em que qualquer termo da nossa proposição puder ser transformado em uma variável, a nossa proposição poderá ser generalizada; e na medida em que isso seja possível, é a incumbência da matemática a fazê-lo. Se tiver várias cadeias de dedução que diferem somente em relação ao significado dos símbolos, de tal forma que proposições simbolicamente idênticas sejam capazes de [receber] várias interpretações, o procedimento apropriado, matematicamente, será o de formar classes de significados que podem aderir aos símbolos, e afirmar que a fórmula em tela se segue da hipótese de que os símbolos pertencem à referida classe. Dessa maneira, símbolos que representavam constantes são transformados em variáveis, e novas constantes, consistindo em classes às quais as velhas classes pertencem, são substituídas. Casos de tais generalizações são tão frequentes que muitos advirão de imediato a todo matemático, e inumeráveis instâncias serão dadas na presente obra. Sempre que dois conjuntos de termos tiveram relações mútuas do mesmo tipo, a mesma forma de dedução aplicará a ambos. Por exemplo, as relações mútuas dos pontos em um plano euclidiano são do mesmo tipo das dos números complexos; logo, a geometria plana, considerada como um ramo da matemática pura, deveria não decidir se suas variáveis sejam pontos ou números complexos ou um outro conjunto de entidades tendo o mesmo tipo de relações mútuas. Em geral, deveríamos lidar, em todo ramo da matemática, com qualquer classe de entidades cujas relações mútuas são de um tipo especificado; assim, a classe, bem como o termo específico considerado, se torna uma variável, e as únicas verdadeiras constantes são os tipos de relações e o que eles envolvem. Ora, tipo de relação significará, na presente discussão, a classe de relações caracterizada pela referida identidade formal das deduções possíveis em relação aos vários membros da classe; e, assim, um tipo de relações, como ficará evidente mais adiante, se não seja já evidente, é sempre uma classe definível em termos de constantes lógicas*15. Podemos, portanto, definir um tipo de relações como uma classe de relações definida por alguma propriedade definível somente em termos de constantes lógicas.

9. Assim, a matemática pura deverá conter nenhum indefinível, exceto constantes lógicas e, consequentemente, nenhuma premissa, ou proposição indemonstrável, exceto as relacionadas exclusivamente com constantes lógicas e com variáveis. É precisamente isso que diferencia a matemática pura da aplicada. Na matemática aplicada, resultados que a matemática pura tem mostrado deduzíveis de alguma hipótese sobre a variável são de fato afirmados de alguma constante satisfazendo a hipótese em tela. Assim, termos que eram variáveis se tornam constantes e uma nova premissa é sempre exigida, a saber: essa entidade específica satisfaz a hipótese em tela. Assim, por exemplo, a Geometria Euclidiana, como um ramo da matemática pura, consiste somente em proposições tendo a hipótese de que "S é um espaço euclidiano". Se acrescentarmos: "O espaço que existe é

\footnotetext{
${ }^{15}$ Nota de Russell: *Um-um, muitos-um, transitivos, simétricos, são instâncias de tipos de relações com os quais nos lidaremos frequentemente.
} 
euclidiano", isso nos permitirá afirmar, do espaço que existe, todos os consequentes de todos os hipotéticos constituindo a Geometria Euclidiana, onde agora a variável $S$ é substituída pela constante espaço real. Mas, por esse passo, vamos da matemática pura à aplicada.

10. A conexão da matemática com a lógica, segundo a explanação [dada] acima, é bastante estreita. $\mathrm{O}$ fato de que todas as constantes matemáticas são constantes lógicas e que todas as premissas matemáticas são envolvidas com estas, dá, acredito eu, a formulação precisa de que os filósofos queriam dizer ao afirmar que a matemática é a priori. De fato, uma vez que a estrutura da lógica tem sido aceita, toda a matemática se segue necessariamente. As próprias constantes lógicas devem ser definidas somente por enumeração, pois são tão fundamentais que todas as propriedades pelas quais a classe delas pudesse ser definida pressupõem alguns termos da classe. Mas, em termos práticos, o método de descobrir as constantes lógicas é a análise da lógica simbólica, que será abordada nos seguintes capítulos. A diferença entre a lógica e a matemática é bastante arbitrária, mas se se quiser [fazer] uma diferença, poder-se-á fazê-la da seguinte maneira. A lógica consiste nas premissas da matemática, juntas com todas as outras proposições que se envolvem exclusivamente com constantes lógicas e com variáveis, mas que não satisfazem a referida definição da matemática (§1). A matemática consiste em todas as consequências das referidas premissas que afirmam implicações formais contendo variáveis, juntas com as, dentro das próprias premissas, que têm essas características. Assim, algumas das premissas da matemática, e.g. o princípio do silogismo, "se $p$ implica $q$ e $q$ implica $r$, então $p$ implica $r$ ”, pertencerão à matemática, enquanto outras, como "implicação é uma relação", pertencerão à lógica, mas não à matemática. Se não fosse pelo desejo de aderir à linguagem costumeira, poderíamos identificar a matemática e a lógica, e definir as duas como a classe de proposições contendo apenas variáveis e constantes lógicas; mas, respeito à tradição me leva, ao contrário, a aderir à referida diferença, enquanto reconheço que certas proposições pertençam a todas as duas ciências. Do que foi dito, o leitor perceberá que a presente obra deveria satisfazer dois objetivos, primeiro, o de mostrar que toda a matemática se segue da lógica simbólica, e, segundo, o de descobrir, na medida do possível, quais são os princípios da própria lógica simbólica. O primeiro desses objetivos será procurado nas seguintes Partes, enquanto o segundo pertence à Parte I. E em primeiro lugar, como um preâmbulo a uma análise crítica, será necessário dar um esboço da Lógica Simbólica, considerada simplesmente como um ramo da matemática. Isso ocupará o seguinte capítulo. 


\section{Anexo II}

[De The Principles of Mathematics ${ }^{16}$ por Bertrand Russell]

\section{CHAPTER I \\ DEFINITION OF PURE MATHEMATICS}

[2] 1. Pure Mathematics is the class of all propositions of the form " $p$ implies $q$," where $p$ and $q$ are propositions containing one or more variables, the same in the two propositions, and neither $p$ nor $q$ contains any constants except logical constants. And logical constants are all notions definable in terms of the following: Implication, the relation of a term to a class of which it is a member, the notion of such that, the notion of relation, and such further notions as may be involved in the general notion of propositions of the above form. In addition to these, mathematics uses a notion which is not a constituent of the propositions which it considers, namely the notion of truth.

2. The above definition of pure mathematics is, no doubt, somewhat unusual. Its various parts, nevertheless, appear to be capable of exact justification-a justification which it will be the object of the present work to provide. It will be shown that whatever has, in the past, been regarded as pure mathematics, is included in our definition, and that whatever else is included possesses those marks by which mathematics is commonly though vaguely distinguished from other studies. The definition professes to be, not an arbitrary decision to use a common word in an uncommon signification, but rather a precise analysis of the ideas which, more or less unconsciously, are implied in the ordinary employment of the term. Our [3] method will therefore be one of analysis, and our problem may be called philosophical-in the sense, that is to say, that we seek to pass from the complex to the simple, from the demonstrable to its indemonstrable premisses. But in one respect not a few of our discussions will differ from those that are usually called philosophical. We shall be able, thanks to the labours of the mathematicians themselves, to arrive at certainty in regard to most of the questions with which we shall be concerned; and among those capable of an exact solution we shall find many of the problems which, in the past, have been involved in all the traditional uncertainty of philosophical strife. The nature of number, of infinity, of space, time and motion, and of mathematical inference itself, are all questions to which, in the present work, an answer professing itself demonstrable with mathematical certainty will be given - an answer which, however, consists in reducing the above problems to problems in pure logic, which last will not be found satisfactorily solved in what follows.

3. The Philosophy of Mathematics has been hitherto as controversial, obscure and unprogressive as the other branches of philosophy. Although it was generally agreed that mathematics is in some sense true, philosophers disputed as to what mathematical propositions really meant: although something was true, no two people were agreed as to

\footnotetext{
${ }^{16} \mathrm{O}$ texto original pode ser acessado no seguinte site: https://people.umass.edu/klement/pom/pom.pdf. Os números entre colchetes indicam a paginação do texto nesse site.
} 
what it was that was true, and if something was known, no one knew what it was that was known. So long, however, as this was doubtful, it could hardly be said that any certain and exact knowledge was to be obtained in mathematics. We find, accordingly, that idealists have tended more and more to regard all mathematics as dealing with mere appearance, while empiricists have held everything mathematical to be approximation to some exact truth about which they had nothing to tell us. This state of things, it must be confessed, was thoroughly unsatisfactory. [4] Philosophy asks of Mathematics: What does it mean? Mathematics in the past was unable to answer, and Philosophy answered by introducing the totally irrelevant notion of mind. But now Mathematics is able to answer, so far at least as to reduce the whole of its propositions to certain fundamental notions of logic. At this point, the discussion must be resumed by Philosophy. I shall endeavour to indicate what are the fundamental notions involved, to prove at length that no others occur in mathematics, and to point out briefly the philosophical difficulties involved in the analysis of these notions. A complete treatment of these difficulties would involve a treatise on Logic, which will not be found in the following pages.

4. There was, until very lately, a special difficulty in the principles of mathematics. It seemed plain that mathematics consists of deductions, and yet the orthodox accounts of deduction were largely or wholly inapplicable to existing mathematics. Not only the Aristotelian syllogistic theory, but also the modern doctrines of Symbolic Logic, were either theoretically inadequate to mathematical reasoning, or at any rate required such artificial forms of statement that they could not be practically applied. In this fact lay the strength of the Kantian view, which asserted that mathematical reasoning is not strictly formal, but always uses intuitions, i.e. the à priori knowledge of space and time. Thanks to the progress of Symbolic Logic, especially as treated by Professor Peano, this part of the Kantian philosophy is now capable of a final and irrevocable refutation. By the help of ten principles of deduction and ten other premisses of a general logical nature (e.g. "implication is a relation"), all mathematics can be strictly and formally deduced; and all the entities that occur in mathematics can be defined in terms of those that occur in the above twenty premisses. In this statement, Mathematics includes not only Arithmetic and Analysis, but also Geometry, Euclidean and non-Euclidean, [5] rational Dynamics, and an indefinite number of other studies still unborn or in their infancy. The fact that all Mathematics is Symbolic Logic is one of the greatest discoveries of our age; and when this fact has been established, the remainder of the principles of mathematics consists in the analysis of Symbolic Logic itself.

5. The general doctrine that all mathematics is deduction by logical principles from logical principles was strongly advocated by Leibniz, who urged constantly that axioms ought to be proved and that all except a few fundamental notions ought to be defined. But owing partly to a faulty logic, partly to belief in the logical necessity of Euclidean Geometry, he was led into hopeless errors in the endeavour to carry out in detail a view which, in its general outline, is now known to be correct*. The actual propositions of Euclid, for

**** On this subject, cf. Couturat, La Logique de Leibniz, Paris, 1901. 
example, do not follow from the principles of logic alone; and the perception of this fact led Kant to his innovations in the theory of knowledge. But since the growth of non-Euclidean Geometry, it has appeared that pure mathematics has no concern with the question whether the axioms and propositions of Euclid hold of actual space or not: this is a question for applied mathematics, to be decided, so far as any decision is possible, by experiment and observation. What pure mathematics asserts is merely that the Euclidean propositions follow from the Euclidean axioms-i.e. it asserts an implication: any space which has such and such properties has also such and such other properties. Thus, as dealt with in pure mathematics, the Euclidean and non-Euclidean Geometries are equally true: in each nothing is affirmed except implications. All propositions as to what actually exists, like the space we live in, belong to experimental or empirical science, not to mathematics; when they belong to applied mathematics, they arise from giv-[6]ing to one or more of the variables in a proposition of pure mathematics some constant value satisfying the hypothesis, and thus enabling us, for that value of the variable, actually to assert both hypothesis and consequent instead of asserting merely the implication. We assert always in mathematics that if a certain assertion $p$ is true of any entity $x$, or of any set of entities $x, y, z, \ldots$, then some other assertion $q$ is true of those entities; but we do not assert either $p$ or $q$ separately of our entities. We assert a relation between the assertions $p$ and $q$, which I shall call formal implication.

6. Mathematical propositions are not only characterized by the fact that they assert implications, but also by the fact that they contain variables. The notion of the variable is one of the most difficult with which Logic has to deal, and in the present work a satisfactory theory as to its nature, in spite of much discussion, will hardly be found. For the present, I only wish to make it plain that there are variables in all mathematical propositions, even where at first sight they might seem to be absent. Elementary Arithmetic might be thought to form an exception: $1+1=2$ appears neither to contain variables nor to assert an implication. But as a matter of fact, as will be shown in Part II, the true meaning of this proposition is: "If $x$ is one and $y$ is one, and $x$ differs from $y$, then $x$ and $y$ are two." And this proposition both contains variables and asserts an implication. We shall find always, in all mathematical propositions, that the words any or some occur; and these words are the marks of a variable and a formal implication. Thus the above proposition may be expressed in the form: "Any unit and any other unit are two units." The typical proposition of mathematics is of the form " $\varphi(x, y, z, \ldots)$ implies $\psi(x, y, z, \ldots)$, whatever values $x, y, z, \ldots$ may have"; where $\varphi(x, y, z, \ldots)$ and $\psi(x, y, z, \ldots)$, for every set of values of $x, y, z, \ldots$, are propositions. It is not asserted that $\varphi$ is always true, nor yet that $\psi$ [7] is always true, but merely that, in all cases, when $\varphi$ is false as much as when $\varphi$ is true, $\psi$ follows from it.

The distinction between a variable and a constant is somewhat obscured by mathematical usage. It is customary, for example, to speak of parameters as in some sense constants, but this is a usage which we shall have to reject. A constant is to be something absolutely definite, concerning which there is no ambiguity whatever. Thus $1,2,3, e, \pi$, Socrates, are constants; and so are man, and the human race, past, present and future, considered collectively. Proposition, implication, class, etc. are constants; but a proposition, 
any proposition, some proposition, are not constants, for these phrases do not denote one definite object. And thus what are called parameters are simply variables. Take, for example, the equation $a x+b y+c=0$, considered as the equation to a straight line in a plane. Here we say that $x$ and $y$ are variables, while $a, b, c$ are constants. But unless we are dealing with one absolutely particular line, say the line from a particular point in London to a particular point in Cambridge, our $a, b, c$ are not definite numbers, but stand for any numbers, and are thus also variables. And in Geometry nobody does deal with actual particular lines; we always discuss any line. The point is that we collect the various couples $x, y$ into classes of classes, each class being defined as those couples that have a certain fixed relation to one triad $(a, b, c)$. But from class to class, $a, b, c$ also vary, and are therefore properly variables.

7. It is customary in mathematics to regard our variables as restricted to certain classes: in Arithmetic, for instance, they are supposed to stand for numbers. But this only means that if they stand for numbers, they satisfy some formula, i.e. the hypothesis that they are numbers implies the formula. This, then, is what is really asserted, and in this proposition it is no longer necessary that our variables should be numbers: the im-[8]plication holds equally when they are not so. Thus, for example, the proposition " $x$ and $y$ are numbers implies $(x+$ $y)^{2}=x^{2}+2 x y+y^{2}$ " holds equally if for $x$ and $y$ we substitute Socrates and Plato* : both hypothesis and consequent, in this case, will be false, but the implication will still be true. Thus in every proposition of pure mathematics, when fully stated, the variables have an absolutely unrestricted field: any conceivable entity may be substituted for any one of our variables without impairing the truth of our proposition.

8. We can now understand why the constants in mathematics are to be restricted to logical constants in the sense defined above. The process of transforming constants in a proposition into variables leads to what is called generalization, and gives us, as it were, the formal essence of a proposition. Mathematics is interested exclusively in types of propositions; if a proposition $p$ containing only constants be proposed, and for a certain one of its terms we imagine others to be successively substituted, the result will in general be sometimes true and sometimes false. Thus, for example, we have "Socrates is a man"; here we turn Socrates into a variable, and consider " $x$ is a man." Some hypotheses as to $x$, for example, " $x$ is a Greek," insure the truth of " $x$ is a man"; thus " $x$ is a Greek" implies " $x$ is a man," and this holds for all values of $x$. But the statement is not one of pure mathematics, because it depends upon the particular nature of Greek and man. We may, however, vary these too, and obtain: If $a$ and $b$ are classes, and $a$ is contained in $b$, then " $x$ is an $a$ " implies " $x$ is a $b$." Here at last we have a proposition of pure mathematics, containing three variables and the constants class, contained in, and those involved in the notion of formal implications with variables. So long as any term in our [9] proposition can be turned into a variable, our proposition can be generalized; and so long as this is possible, it is the business of mathematics to do it. If there are several chains of deduction which differ only as to the

$* * * *$ It is necessary to suppose arithmetical addition and multiplication defined (as may be easily done) so that the above formula remains significant when $x$ and $y$ are not numbers.

RBHM, Vol. 21, n 42, pp. 329-349, 2021 
meaning of the symbols, so that propositions symbolically identical become capable of several interpretations, the proper course, mathematically, is to form the class of meanings which may attach to the symbols, and to assert that the formula in question follows from the hypothesis that the symbols belong to the class in question. In this way, symbols which stood for constants become transformed into variables, and new constants are substituted, consisting of classes to which the old constants belong. Cases of such generalization are so frequent that many will occur at once to every mathematician, and innumerable instances will be given in the present work. Whenever two sets of terms have mutual relations of the same type, the same form of deduction will apply to both. For example, the mutual relations of points in a Euclidean plane are of the same type as those of the complex numbers; hence plane geometry, considered as a branch of pure mathematics, ought not to decide whether its variables are points or complex numbers or some other set of entities having the same type of mutual relations. Speaking generally, we ought to deal, in every branch of mathematics, with any class of entities whose mutual relations are of a specified type; thus the class, as well as the particular term considered, becomes a variable, and the only true constants are the types of relations and what they involve. Now a type of relation is to mean, in this discussion, a class of relations characterized by the above formal identity of the deductions possible in regard to the various members of the class; and hence a type of relations, as will appear more fully hereafter, if not already evident, is always a class definable in terms of logical constants*. [10] We may therefore define a type of relations as a class of relations defined by some property definable in terms of logical constants alone.

9. Thus pure mathematics must contain no indefinables except logical constants, and consequently no premisses, or indemonstrable propositions, but such as are concerned exclusively with logical constants and with variables. It is precisely this that distinguishes pure from applied mathematics. In applied mathematics, results which have been shown by pure mathematics to follow from some hypothesis as to the variable are actually asserted of some constant satisfying the hypothesis in question. Thus terms which were variables become constant, and a new premiss is always required, namely: this particular entity satisfies the hypothesis in question. Thus for example Euclidean Geometry, as a branch of pure mathematics, consists wholly of propositions having the hypothesis " $S$ is a Euclidean space." If we go on to: "The space that exists is Euclidean," this enables us to assert of the space that exists the consequents of all the hypotheticals constituting Euclidean Geometry, where now the variable $S$ is replaced by the constant actual space. But by this step we pass from pure to applied mathematics.

10. The connection of mathematics with logic, according to the above account, is exceedingly close. The fact that all mathematical constants are logical constants, and that all the premisses of mathematics are concerned with these, gives, I believe, the precise statement of what philosophers have meant in asserting that mathematics is à priori. The fact is that, when once the apparatus of logic has been accepted, all mathematics necessarily

$* * * *$ One-one, many-one, transitive, symmetrical, are instances of types of relations with which we shall be often concerned. 
follows. The logical constants themselves are to be defined only by enumeration, for they are so fundamental that all the properties by which the class of them might be [11]

defined presuppose some terms of the class. But practically, the method of discovering the logical constants is the analysis of symbolic logic, which will be the business of the following chapters. The distinction of mathematics from logic is very arbitrary, but if a distinction is desired, it may be made as follows. Logic consists of the premisses of mathematics, together with all other propositions which are concerned exclusively with logical constants and with variables but do not fulfil the above definition of mathematics (§1). Mathematics consists of all the consequences of the above premisses which assert formal implications containing variables, together with such of the premisses themselves as have these marks. Thus some of the premisses of mathematics, e.g. the principle of the syllogism, "if $p$ implies $q$ and $q$ implies $r$, then $p$ implies $r$," will belong to mathematics, while others, such as "implication is a relation," will belong to logic but not to mathematics. But for the desire to adhere to usage, we might identify mathematics and logic, and define either as the class of propositions containing only variables and logical constants; but respect for tradition leads me rather to adhere to the above distinction, while recognizing that certain propositions belong to both sciences. From what has now been said, the reader will perceive that the present work has to fulfil two objects, first, to show that all mathematics follows from symbolic logic, and secondly to discover, as far as possible, what are the principles of symbolic logic itself. The first of these objects will be pursued in the following Parts, while the second belongs to Part I. And first of all, as a preliminary to a critical analysis, it will be necessary to give an outline of Symbolic Logic considered simply as a branch of mathematics. This will occupy the following chapter. 